\title{
Influence of cardiac motion on diffusion-weighted magnetic resonance imaging of the liver
}

\author{
Thomas C. Kwee - Taro Takahara • Tetsu Niwa • \\ Marko K. Ivancevic • Gwenael Herigault • \\ Marc Van Cauteren • Peter R. Luijten
}

Received: 1 June 2009 / Revised: 3 August 2009 / Accepted: 12 August 2009 / Published online: 2 September 2009

(C) The Author(s) 2009. This article is published with open access at Springerlink.com

\begin{abstract}
Purpose To assess cardiac motion-induced signal loss in diffusion-weighted magnetic resonance imaging (DWI) of the liver using dynamic DWI.

Materials and methods Three volunteers underwent dynamic coronal DWI of the liver under breathholding, in the diastolic $\left(\mathrm{DWI}_{\text {diast }}\right)$ or systolic $\left(\mathrm{DWI}_{\text {syst }}\right)$ cardiac phase, and with motion probing gradients (MPGs) in phase encoding ( $\mathrm{P}$, left-right), frequency encoding (M, superior-inferior), or slice select ( $\mathrm{S}$, anterior-posterior) direction. Liver-to-background contrasts (LBCs) of DWI $\mathrm{Dyst}_{\text {st }}$ were compared to those of $\mathrm{DWI}_{\text {diast }}$, for both the left and right liver lobes, using nonparametric tests. Signal decrease ratios (SDRs) were calculated as $\left(1-\left(\mathrm{LBC} \mathrm{DWI}_{\text {syst }} / \mathrm{LBC} \mathrm{DWI}_{\text {diast }}\right)\right) \times 100 \%$. DWI $\mathrm{I}_{\text {syst }}$
\end{abstract}

T. C. Kwee $(\varangle) \cdot$ T. Takahara · T. Niwa · P. R. Luijten Department of Radiology, University Medical Center Utrecht, Heidelberglaan 100, 3584 CX Utrecht, The Netherlands e-mail: thomaskwee@gmail.com

T. Niwa

Department of Radiology,

Kanagawa Children's Medical Center,

Yokohama, Japan

M. K. Ivancevic

Department of Radiology,

University of Michigan Medical Center,

Ann Arbor, MI, USA

M. K. Ivancevic

Philips Healthcare, Cleveland, OH, USA

G. Herigault

Philips Healthcare, Best, The Netherlands

M. Van Cauteren

Philips Healthcare Asia Pacific, Tokyo, Japan was further analyzed to determine which direction of MPGs was most affected by cardiac motion.

Results In the left liver lobe, LBCs of $\mathrm{DWI}_{\text {syst }}$ (median $3.35)$ were significantly lower $(P<0.0001)$ than those of DWI $\mathrm{D}_{\text {diast }}$ (median 4.84). In the right liver lobe, LBCs of $\mathrm{DWI}_{\text {syst }}$ (median 4.17) were also significantly lower $(P<$ 0.0001) than those of DWI diast (median 5.35). SDRs of the left and right liver lobes were $25.5 \%$ and $17.3 \%$, respectively. In $\mathrm{DWI}_{\text {syst }}$, the significantly lowest $(P<0.05)$ LBCs were observed in the $\mathrm{M}$ direction (left liver lobe) and $\mathrm{P}$ direction (right liver lobe) of MPGs.

Conclusion Signal intensity of both liver lobes are affected by cardiac motion in DWI. In the left liver lobe, signal loss especially occurs in the superior-inferior direction of MPGs, whereas in the right lobe, signal loss especially occurs in the left-right direction of MPGs.

Keywords Diffusion-weighted magnetic resonance imaging · DWI · Dynamic · Liver - Cardiac motion · Pseudo-anisotropy artifact

\section{Introduction}

Diffusion-weighted magnetic resonance (MR) imaging (DWI) offers high lesion-to-background contrast and allows quantifying diffusion by means of apparent diffusion coefficient (ADC) measurements, making it a valuable technique for the detection and characterization of liver lesions [1,2], and characterization of the liver parenchyma itself $[3,4]$. However, signal loss in DWI and artificially elevated ADCs have been reported to occur in the left liver lobe [5-7]. A possible explanation for this phenomenon is cardiac motion. Cardiac motion during the systolic phase may shake or distort the left liver lobe which is located just below the heart. 
Consequently, regional deformation and/or acceleration may occur, which affects intravoxel incoherent motion imaging and measurements (i.e. DWI and ADC measurements, respectively) [5-7]. Thus, if no cardiac triggering is employed, both detectability of lesions, and reliability and reproducibility of ADC measurements in the left lobe of the liver may be impaired [5-7]. On the other hand, it has recently been reported that signal loss and artificially elevated ADCs may be encountered in the right liver lobe as well [8]. This artifact was observed in DWI under respiratory triggering and was speculated to originate from respiratory motion [8]. However, it can be hypothesized that cardiac motion may also play a causative role in the occurrence of this artifact in the right lobe of the liver. The purpose of this study was therefore to determine if, and to what extent, cardiac motion causes signal loss in DWI of the liver, with special focus on the right liver lobe. To examine the effects of the heart on the liver in DWI, we developed a new sequence to acquire dynamic diffusion-weighted images.

\section{Materials and methods}

\section{Study participants}

This study was approved by the local Institutional Review Board and written informed consent was obtained from all participants. Three healthy adult volunteers (one men and two women aged 28, 24, and 29 years, respectively) with no prior history or findings related to liver disease at the time of the study prospectively underwent DWI of the liver. Exclusion criteria were general contraindications to MR imaging, such as implanted pacemaker and claustrophobia.

\section{MR imaging}

All volunteers were examined with a $1.5 \mathrm{~T}$ MR scanner (Achieva, Philips Healthcare, Best, The Netherlands) using a 16-element phased-array surface coil (SENSE XL Torso coil, Philips Healthcare). Dynamic DWI was performed using a single-shot spin-echo echo-planar imaging (EPI) sequence with the following parameters: eight dynamics, repetition time of three heart beats, echo time of $70 \mathrm{~ms}$, image acquisition in the coronal plane, slice thickness of $10 \mathrm{~mm}$, singlesection acquisition (this single slice included both the left and right liver lobes, and the middle hepatic vein), field of view of $360 \times 405 \mathrm{~mm}^{2}$, acquisition matrix of $128 \times 115$, acquired voxel size of $2.81 \times 3.50 \times 10.00 \mathrm{~mm}^{3}$, reconstructed voxel size of $1.41 \times 1.41 \times 10.00 \mathrm{~mm}^{3}$, parallel acceleration [SENSitivity Encoding (SENSE)] factor of 2, EPI factor of 61, motion probing gradients (MPGs) in one direction [phase encoding ( $\mathrm{P}$, left-right), frequency encoding (M, superior-inferior), or slice select ( $\mathrm{S}$, anterior-posterior)], $b$-value of $500 \mathrm{~s} / \mathrm{mm}^{2}$, number of excitations of 1 , partial Fourier acquisition (half scan factor) of $60 \%$, spectral presaturation inversion recovery (SPIR) fat suppression, breathhold image acquisition (in order to minimize bias from respiratory motion), finger pulse triggering [to the systolic heart phase $\left(\mathrm{DWI}_{\text {syst }}\right.$, trigger delay of $27.4 \mathrm{~ms}$ ) or to the diastolic heart phase $\left(\mathrm{DWI}_{\text {diast }}\right.$, trigger delay varying between 400 and $600 \mathrm{~ms}$, depending on the heart rate)] [5], and nominal scan time of $33 \mathrm{~s}$ [for 8 dynamics acquired with one direction of MPGs (P, M, or S) in one cardiac phase (systolic or diastolic)]. In each volunteer, three series of eight dynamics were obtained for $\mathrm{P}, \mathrm{M}$, and $\mathrm{S}$ directions of MPGs separately, and for the systolic and diastolic heart phases separately, yielding a total of 144 dynamics per subject. Of note, before starting the actual experiment, optimum trigger delay for diastolic scanning in each volunteer was determined by increasing trigger delay in steps of $100 \mathrm{~ms}$ between 300 and $700 \mathrm{~ms}$, and selecting the trigger delay which yielded the maximum signal intensity of the heart. In two volunteers with heart rates of 50 and 60 beats/min an optimum trigger delay of $600 \mathrm{~ms}$ was applied, while in the third volunteer with a heart rate of 80 beats/min an optimum trigger delay of $400 \mathrm{~ms}$ was applied for each dynamic that was acquired in the diastolic phase, which is in line with previously reported data by Mürtz et al. [5]. Quality of breathholding and pulse triggering in each acquisition were checked by the operator on the monitoring screen and, if deemed of insufficient quality, the sequence was repeated.

Image analysis

The liver was divided into a left and a right liver lobe using the middle hepatic vein as an anatomical reference. Liverto-background contrasts of the left and right liver lobe were measured by dividing signal intensity of a region of interest (ROI) encompassing the left or right liver lobe by the standard deviation of the signal intensity of a rectangular-shaped $\mathrm{ROI}$ in the lung. The latter was placed in the upper part of the lung, above and excluding the heart (Fig. 1); this approach was chosen because it was not possible to calculate a real signal-to-noise ratio by placing a ROI outside the body due to the use of SENSE.

\section{Statistical analysis}

LBCs of the left lobe in the systolic phase and LBCs of the right lobe in the diastolic phase and were not normally distributed (Kolmogorov-Smirnov test, $P=0.026$ and 0.029 , respectively). $\mathrm{LBCs}$ of $\mathrm{DWI}_{\text {syst }}$ were compared to those of $\mathrm{DWI}_{\text {diast }}$, for the left and right liver lobe separately, by using the nonparametric Mann-Whitney $U$ test. In addition, signal decrease ratios (SDRs) were calculated as $(1-$ (LBC $\left.\left.\mathrm{DWI}_{\text {syst }} / \mathrm{LBC} \mathrm{DWI}_{\text {diast }}\right)\right) \times 100 \%$. Subsequently, $\mathrm{BH}_{\text {syst }}$ was 


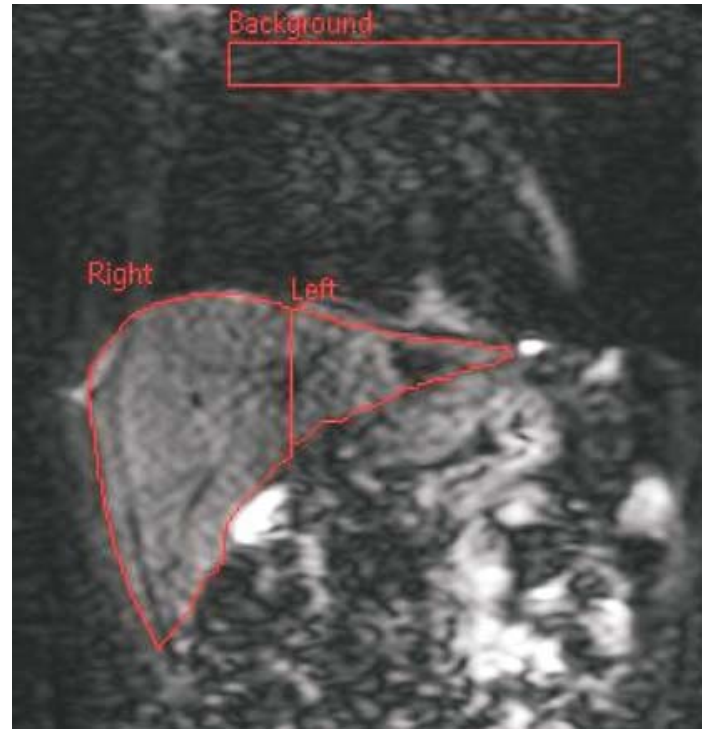

Fig. 1 Example of regions of interest placed in the left liver lobe (left), right liver lobe (right), and lung (background)

further analyzed to determine which direction of MPGs was most affected by cardiac motion, for the left and right liver lobe separately. To that end, DWI $\mathrm{Dyst}_{\text {st }}$ scans with MPGs in $\mathrm{M}, \mathrm{P}$, and $\mathrm{S}$ directions were compared by using the nonparametric Kruskal-Wallis test followed by post-hoc pairwise comparisons according to Conover [9]. $P$ values less than 0.05 were considered to indicate statistically significant differences. Statistical analyses were executed using MedCalc Software (MedCalc, Mariakerke, Belgium).

\section{Results}

In the left liver lobe, $\mathrm{LBCs}_{\mathrm{S}}$ of $\mathrm{DWI}_{\text {syst }}$ (median 3.35, range 1.09-6.32) were significantly lower $(P<0.0001)$ than those of DWI $I_{\text {diast }}$ (median 4.84, range 1.19-7.06). Interestingly, in the right liver lobe, $\mathrm{LBCs}_{\text {of }} \mathrm{DWI}_{\text {syst }}$ (median 4.17, range $1.39-8.04)$ were also significantly lower $(P<0.0001)$ than those of $\mathrm{DWI}_{\text {diast }}$ (median 5.35, range 1.85-7.67). SDRs of the left and right liver lobes were 25.5 and $17.3 \%$, respectively.

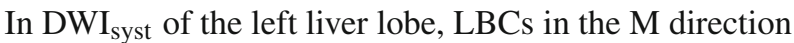
of MPGs (median 2.30, range 1.09-5.21) were significantly lower $(P<0.05)$ than those in the $\mathrm{P}$ (median 3.60, range 1.76-5.57) and $\mathrm{S}$ (median 4.45, range 2.05-6.32) directions of MPGs. In addition, LBCs in the $\mathrm{P}$ and $\mathrm{S}$ directions of MPGs were also significantly different $(P<0.05)$ (Fig. 2). On the other hand, in DWI $\mathrm{Syst}_{\mathrm{st}}$ of the right liver lobe, LBCs in the $\mathrm{P}$ direction of MPGs (median 3.81, range 1.39-6.04) were significantly lower $(P<0.05)$ than those in the $\mathrm{M}$ (median 4.34, range 1.97-6.97) and $\mathrm{S}$ (median 4.53, range

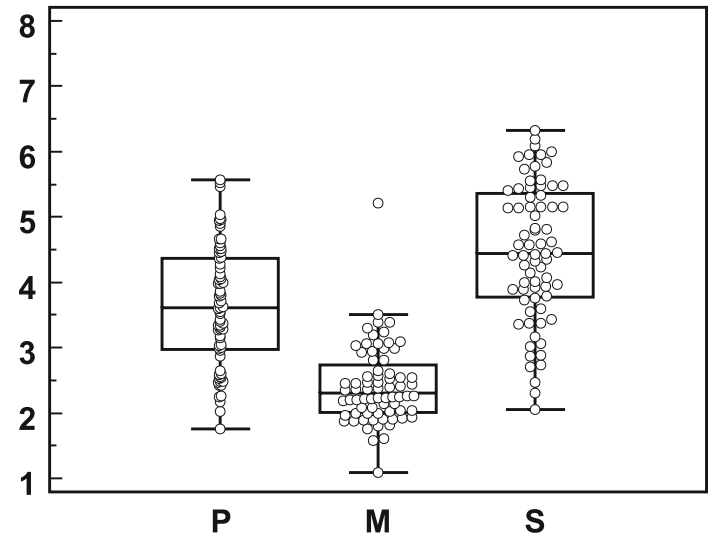

Fig. 2 Box-and-whisker plot of LBCs (including all individual data, plotted as open dots) of the left liver lobe according to $\mathrm{P}, \mathrm{M}$, and $\mathrm{S}$ directions of MPGs, which corresponded to the left-right, superior-inferior, and anterior-posterior directions, respectively, in the present study

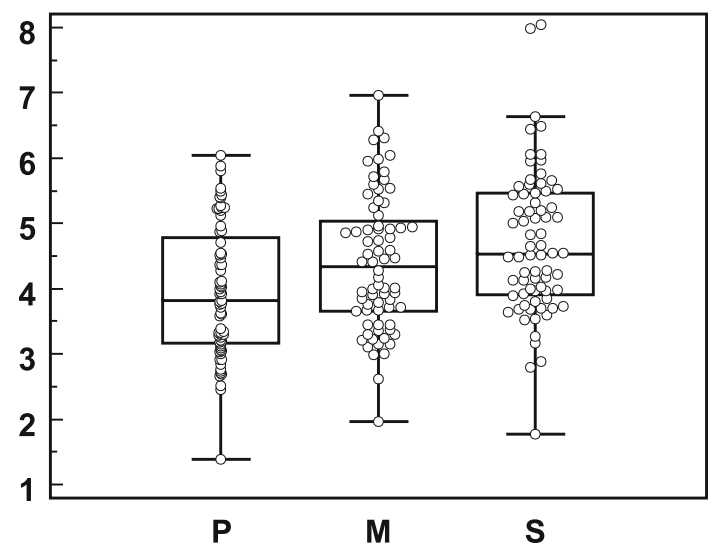

Fig. 3 Box-and-whisker plot of LBCs (including all individual data, plotted as open dots) of the right liver lobe according to P, M, and S directions of MPGs, which corresponded to the left-right, superiorinferior, and anterior-posterior directions, respectively, in the present study

1.77-8.04) directions of MPGs, without any significant difference between M and S directions of MPGs (Fig. 3).

Representative examples are shown in Figs. 4 and 5.

\section{Discussion}

Several studies [5-7] have suggested that signal loss in DWI and artificially increased ADCs can be observed in the left liver lobe due to cardiac motion. In addition, localized signal loss in DWI of the right liver lobe was recently reported to occur in respiratory triggered DWI, depending on difference of directions of MPGs [8]. Nasu et al. [8] speculated that the liver does not remain still, even at the end of the expiratory phase, and may show localized movement such as expansion, contraction and rotation. Consequently, intravoxel deformation and/or acceleration of the liver may 

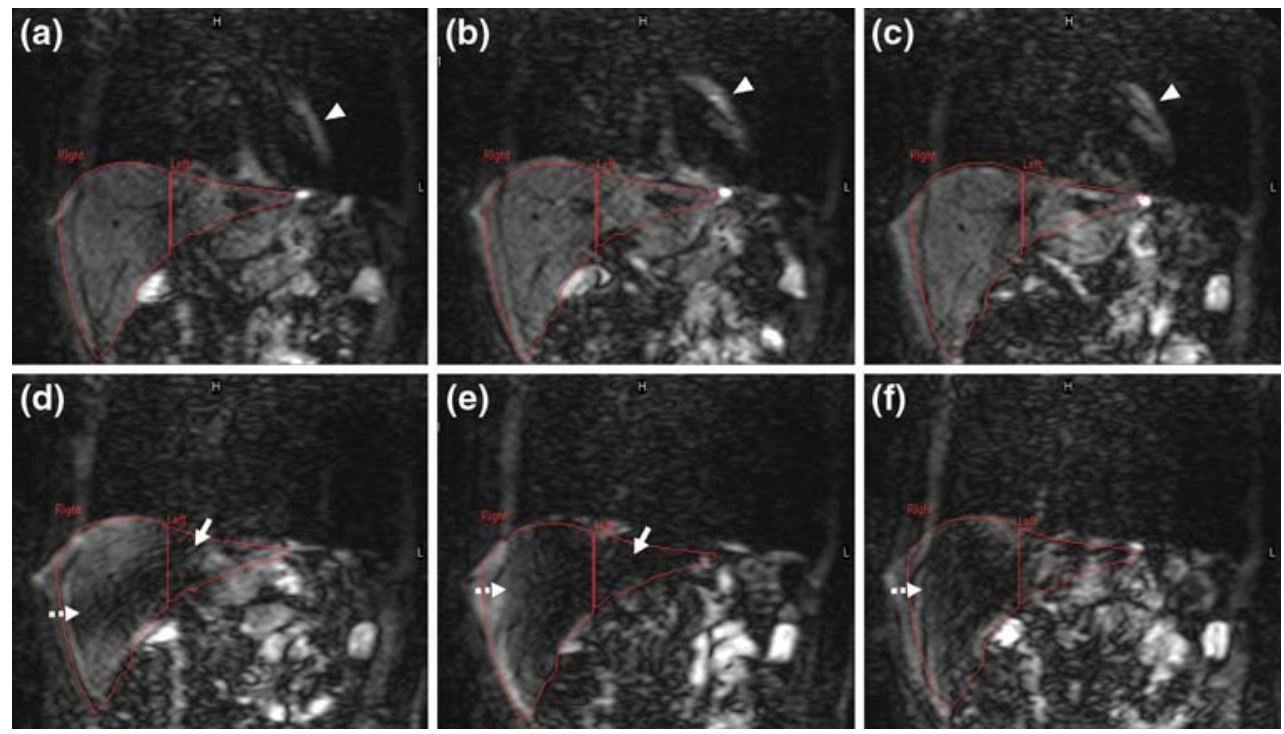

Fig. 4 Dynamic coronal single-section diffusion-weighted images in the diastolic phase (acquired using a trigger delay of $600 \mathrm{~ms}$ ) (a-c) and systolic phase (acquired using a shortest possible trigger delay of $27.4 \mathrm{~ms})(\mathbf{d}-\mathbf{f})$, in $\mathrm{P}(\mathbf{a}, \mathbf{d}$ left-right $), \mathrm{M}$ (b, e superior-inferior), and $\mathrm{S}$ (d, f anterior-posterior) directions of MPGs, in a 28 -year-old male volunteer. In the diastolic phase, no apparent signal loss is observed in both the left and right liver lobes $(\mathbf{a}-\mathbf{c})$. Note the high signal intensity of the heart in the diastolic phase (a-c arrowheads). On the other hand, in the systolic phase, considerable signal loss in both the left (continuous arrows) and right liver lobes (dashed arrows) can be seen (d-f) lead to signal loss in DWI of the right liver lobe and artificially increased ADCs. The recently introduced concept of "diffusion-weighted imaging under split breath-hold acquisition and post-processing" (DWI-SBAP), which is a modified breathhold DWI technique, has shown to significantly reduce signal loss in the right liver lobe compared to respiratory triggered DWI [10]. However, the exact cause(s) of this artifact have not been identified yet.

In the present study, we used dynamic coronal DWI to examine the effects of the heart on the liver in DWI. Advantages of dynamic coronal DWI over axial scanning are the visualization of the liver in the superior-inferior axis (which is the main direction of impact of the heart on the liver), and the availability of a coronal image yielding an overview of both the left and right liver lobes. Furthermore, it enables acquiring dynamic diffusion-weighted images under identical tuning conditions, which yields more reliable data than would be obtained using multiple repetitive breathhold scans. Our results confirm that signal intensity in DWI of the left liver lobe is affected by cardiac motion. More importantly, our results also indicate that signal intensity in DWI of the right liver lobe is also affected by cardiac motion. SDRs of the left and right liver lobes were 25.5 and $17.3 \%$, respectively. Thus, although the right liver lobe suffers less from signal loss than the left liver lobe, signal loss in the right liver lobe is still considerable. There may be several reasons for the observed signal loss. First, direct impact of the heart on the liver may result in non-rigid body motion of both liver lobes. Second, arterial pulsation immediately post-systole may result in the propagation of a compression wave, again leading to non-rigid body motion of both liver lobes, that may extend well beyond the heart; it is well known that this mechanism occurs in (DWI of) the brain [11,12]. Non-rigid body motion may give rise to phase dispersion and signal loss in DWI [12]. Previous work by Robson and Porter [13] indicated that the method of reconstruction may also play a role in the degree of signal loss in non-cardiac-gated DWI of the brain (note that signal loss in DWI of the brain is mainly a result of pulsatile blood flow, rather than direct impact of the heart on this organ). Investigating the interplay between cardiac motion, signal loss, and different reconstruction methods, however, was beyond the scope of the present study. Robson and Porter [13] reported that the use of zero-padded reconstruction methods minimizes the artifacts in non-cardiac-gated acquisitions when compared to the Margosian reconstruction approach. Of note, in the present study zero-padded reconstruction was applied for all acquisitions. Nevertheless, DWI in the systolic phase suffered from more signal loss than DWI in the diastolic phase, making cardiac motion a very likely cause of the observed signal loss, regardless of reconstruction method. It may also be argued that partial Fourier encoding may cause some degree of signal loss. However, it is unlikely that central k-space line is missed, because a partial Fourier acquisition of $60 \%$ was used. Furthermore, although partial Fourier encoding reduces signalto-noise ratio (SNR), this occurs in the whole image, and not 
Fig. 5 Five consecutive dynamic coronal single-section diffusion-weighted images in the systolic phase (acquired using a shortest possible trigger delay of $27.4 \mathrm{~ms})$, in

$\mathrm{P}(P$, left-right $)$,

$\mathrm{M}(M$, superior-inferior $)$, and

$\mathrm{S}$ (S, anterior-posterior) directions of MPGs, in a 24-year-old female volunteer. Note signal loss in both the left (continuous arrows) and right liver lobes (dashed arrows). Signal loss in the right liver lobe is especially seen in the $\mathrm{P}$ direction of MPGs Signal loss in the left liver lobe is most pronounced in the $\mathrm{M}$ direction of MPGs. On the other hand, in the $\mathrm{S}$ direction of MPGs, signal intensity in both the left and right liver lobes is generally well preserved

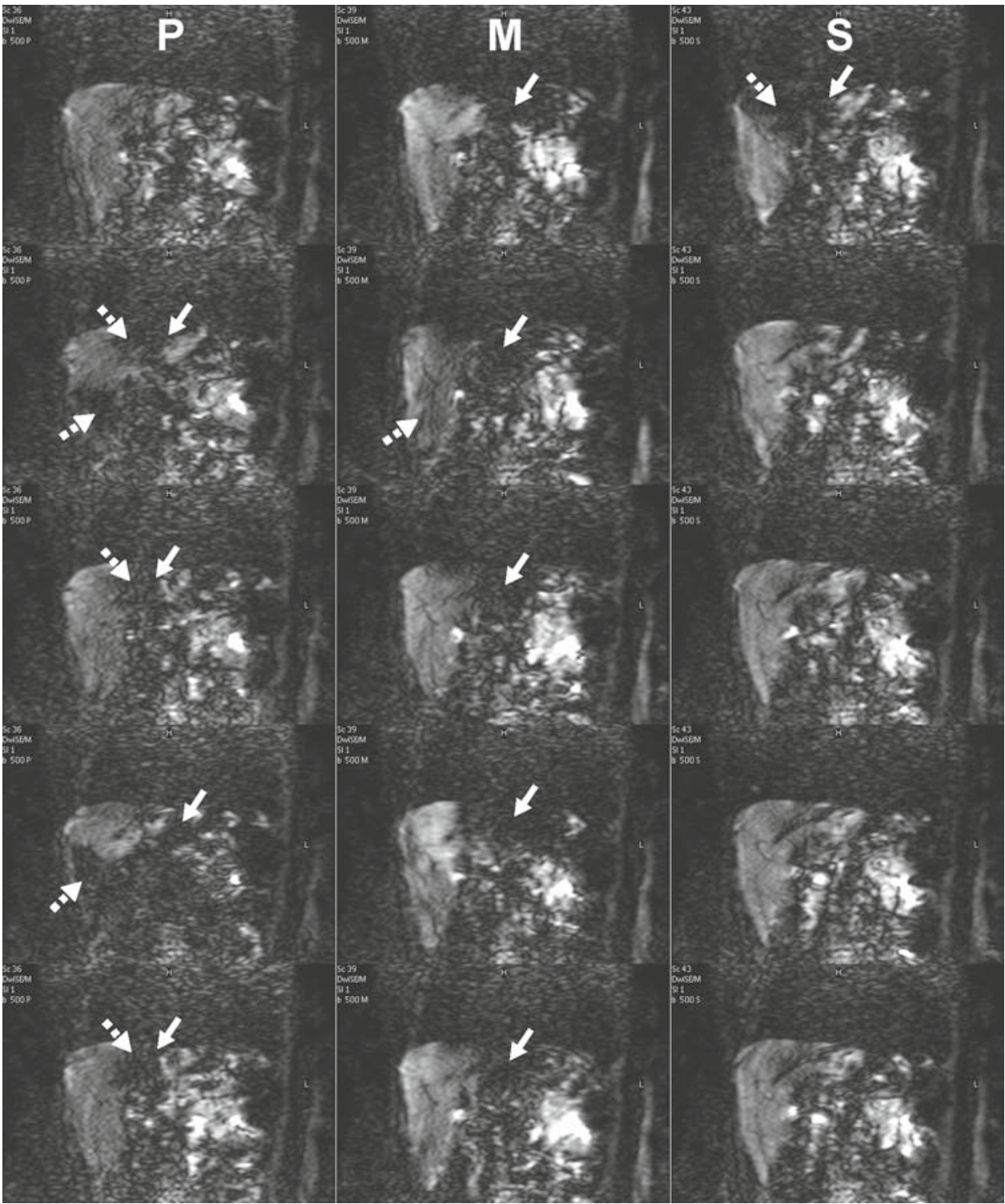

only in the liver. Moreover, the same partial Fourier encoding factor was applied for both systolic and diastolic scans.

Furthermore, in the left liver lobe, signal loss in the $\mathrm{M}$ direction of MPGs (i.e. superior-inferior direction in the present study) due to cardiac motion was significantly higher than that in the P and S directions of MPGs. This finding can be explained by the fact that the highest impact of the heart on the left liver lobe is in the superior-inferior direction, and is consistent with previously reported data [5]. In addition, in the right lobe, signal loss due to cardiac motion is highest in the P direction of MPGs (i.e. left-right direction in the present study), in contrast to the M direction of MPGs (i.e. superior-inferior direction in the present study) in the left lobe. Possibly, the left liver lobe transfers the impact of the heart to the right liver lobe in a mainly lateral direction (i.e. from medial to lateral). Since the $\mathrm{S}$ direction of
MPGs (i.e. anterior-posterior direction in the present study) was the direction that suffered the least from signal loss in both the left and right liver lobes (Figs. 2, 3, 5), it may be a good option to perform DWI with MPGs only in the anterior-posterior direction. This approach may be considered acceptable, since both the liver parenchyma and liver lesions have shown to exhibit diffusion isotropy [14]. Nevertheless, further research should confirm this hypothesis.

Our findings may have important clinical consequences. It may be assumed that cardiac motion artifacts have an impact not only on the signal intensity of the liver parenchyma, but also on the signal intensity of focal liver lesions. Therefore, cardiac motion-induced signal loss may decrease detectability of liver lesions. Second, cardiac motion artifacts may impair reliability and reproducibility of ADC measurements of liver lesions and liver parenchyma. These negative effects 
on qualitative and quantitative evaluation in DWI may be seen in both the left and right liver lobes.

This study had several limitations. First, only one $b$-value $\left(500 \mathrm{~s} / \mathrm{mm}^{2}\right)$ was used, as a result of which no ADC measurements could be performed. However, acquiring one more $b$ value would be impossible because of the use of (pulse-triggered) dynamic DWI and the limited period of breathholding. Furthermore, in the present study, the conventional SNR could not be calculated for each liver lobe, because the standard deviation of the background could not be measured easily because diffusion-weighted images were obtained using SENSE (SNR varies from pixel to pixel as determined by the geometry factor and the regularisation algorithm will force the signal intensity and standard deviation of pixels outside the body to be zero). Therefore, we chose to calculate an LBC, which was defined as signal intensity of the liver (either left or right lobe) divided by the standard deviation of the signal intensity of a rectangular-shaped ROI in the lung. Although suboptimal, in the current setting, the upper part of the lung was the best available location to assess background signal. Another study limitation is that only one excitation could be used for dynamic DWI. Although this is a common approach for breathhold DWI, respiratory triggered and free breathing DWI are usually acquired using multiple excitations. Using a high number of excitations, the probability of data acquisition during the diastolic phase may increase. This, in turn, may decrease signal loss and improve reliability and reproducibility of ADC measurements. Therefore, although it may be stated that pulse triggering is required to improve DWI of the liver and to allow more reliable ADC measurements in breathhold DWI acquired with only one excitation [5], it is still unclear whether cardiac motion compensation techniques are necessary in respiratory triggered and free breathing DWI using a high number of excitations. Another issue is that applied slice thickness in the present study was relatively large $(10 \mathrm{~mm})$, which increased sensitivity of the experiment to intravoxel incoherent motion. In a clinical setting applied slice thickness for DWI is usually smaller. However, the chosen slice thickness offered the best compromise between SNR and sensitivity to diffusion. Furthermore, phase encoding was done in the left-right direction because this is the most favourable direction in direct coronal scanning. Direct coronal scanning, however, offered the advantage of visualizing both liver lobes in a single image in the superior-inferior axis, as mentioned previously. Finally, the present study did not assess the effect of respiratory motion on signal intensity in DWI of the liver. Rather, all DWI scans were performed under breathholding, in order to minimize bias from respiratory motion. However, since respiratory motion may well be another causative factor for signal loss in DWI of the liver, as was suggested by previous studies $[7,8,10]$, further research on this issue is required. Of note, a recent study [15] showed that ADCs of the right liver lobe in axial respiratory triggered DWI (acquired with two excitations) were significantly higher and less reproducible than those in breathhold DWI (acquired with two excitations) and free-breathing DWI (acquired with four and five excitations). Probably a complex interplay between cardiac motion, respiratory motion and DWI acquisition technique determines signal loss in DWI of the liver.

\section{Conclusion}

In conclusion, the results of this study show that dynamic DWI of the liver is feasible. Signal intensity of both the left and right liver lobes are considerably affected by cardiac motion in DWI. In the left liver lobe, signal loss especially occurs in the superior-inferior direction of MPGs, whereas in the right lobe, signal loss especially occurs in the left-right direction of MPGs.

Acknowledgments This work was partly funded by a grant of the Dutch scientific foundation (NWO) for T.T.

Open Access This article is distributed under the terms of the Creative Commons Attribution Noncommercial License which permits any noncommercial use, distribution, and reproduction in any medium, provided the original author(s) and source are credited.

\section{References}

1. Naganawa S, Kawai H, Fukatsu H, Sakurai Y, Aoki I, Miura S, Mimura T, Kanazawa H, Ishigaki T (2005) Diffusion-weighted imaging of the liver: technical challenges and prospects for the future. Magn Reson Med Sci 4:175-186

2. Low RN (2007) Abdominal MRI advances in the detection of liver tumours and characterization. Lancet Oncol 8:525-535

3. Taouli B, Tolia AJ, Losada M, Babb JS, Chan ES, Bannan MA, Tobias H (2007) Diffusion-weighted MRI for quantification of liver fibrosis: preliminary experience. AJR Am J Roentgenol 189:799-806

4. Koinuma M, Ohashi I, Hanafusa K, Shibuya H (2005) Apparent diffusion coefficient measurements with diffusion-weighted magnetic resonance imaging for evaluation of hepatic fibrosis. J Magn Reson Imaging 22:80-85

5. Mürtz P, Flacke S, Träber F, van den Brink JS, Gieseke J, Schild $\mathrm{HH}$ (2002) Abdomen: diffusion-weighted MR imaging with pulsetriggered single-shot sequences. Radiology 224:258-264

6. Nasu K, Kuroki Y, Sekiguchi R, Kazama T, Nakajima H (2006) Measurement of the apparent diffusion coefficient in the liver: is it a reliable index for hepatic disease diagnosis?. Radiat Med 24:438-444

7. Kandpal H, Sharma R, Madhusudhan KS, Kapoor KS (2009) Respiratory-triggered versus breath-hold diffusionweighted MRI of liver lesions: comparison of image quality and apparent diffusion coefficient values. AJR Am J Roentgenol 192:915-922

8. Nasu K, Kuroki Y, Fujii H, Minami M (2007) Hepatic pseudoanisotropy: a specific artifact in hepatic diffusion-weighted images obtained with respiratory triggering. MAGMA 20:205-211

9. Conover WJ (1999) Practical nonparametric statistics. Wiley, New York 
10. Nasu K, Kuroki Y, Minami M (2009) Feasibility of diffusion-weighted imaging under split breath-hold acquisition and postprocessing (DWI-SBAP): an attempt to suppress hepatic pseudo-anisotropy. Jpn J Radiol 27:78-85

11. Greitz D, Wirestam R, Franck A, Nordell B, Thomsen C, Ståhlberg F(1992) Pulsatile brain movement and associated hydrodynamics studied by magnetic resonance phase imaging. The Monro-Kellie doctrine revisited. Neuroradiology 34:370-380

12. Norris DG (2001) Implications of bulk motion for diffusionweighted imaging experiments: effects, mechanisms, and solutions. J Magn Reson Imaging 13:486-495

13. Robson MD, Porter DA (2005) Reconstruction as a source of artifact in non-gated single-shot diffusion-weighted EPI. Magn Reson Imaging 23:899-905
14. Taouli B, Vilgrain V, Dumont E, Daire JL, Fan B, Menu Y (2003) Evaluation of liver diffusion isotropy and characterization of focal hepatic lesions with two single-shot echo-planar MR imaging sequences: prospective study in 66 patients. Radiology 226:7178

15. Kwee TC, Takahara T, Koh DM, Nievelstein RA, Luijten PR (2008) Comparison and reproducibility of ADC measurements in breathhold, respiratory triggered, and free-breathing diffusionweighted MR imaging of the liver. J Magn Reson Imaging 28: $1141-1148$ 